\title{
A case for genotype-guided pain management
}

\author{
Larisa H Cavallari*,1 \& Julie A Johnson ${ }^{1}$ \\ ${ }^{1}$ Department of Pharmacotherapy \& Translational Research, The Center for Pharmacogenomics \& Precision Medicine, University of \\ Florida, Gainesville, FL 32610, USA \\ *Author for correspondence: Tel: +1 352273 8245; Fax: +1 352273 6485; Icavallari@cop.ufl.edu
}

\begin{abstract}
"A CYP2D6-guided approach to pain management, with avoidance of codeine and tramadol in PMs and IMs, can improve pain control, as we showed in our chronic pain study"
\end{abstract}

First draft submitted: 5 May 2019; Accepted for publication: 24 May 2019; Published online: 1 August 2019

Keywords: CYP2D6 • genotype $\bullet$ opioid $\bullet$ pharmacogenetics $\bullet$ precision medicine

\section{Burden of pain}

Pain is a leading cause of disability worldwide and is associated with lost workplace productivity, reduced quality of life, anxiety and depression $[1,2,3]$. Approximately $20 \%$ of US adults suffer from chronic pain, and $8 \%$ suffer from high impact chronic pain, defined as pain that limits life or work activities [2]. Opioids are commonly prescribed to treat pain, with a significant increase in opioid prescribing over the past decade, especially in primary care settings [4]. This has been accompanied by an increase in opioid misuse and opioid-related deaths. In the USA alone, from 1999 to 2017, there were nearly 218,000 deaths from overdose related to prescription opioids [5]. Seeking relief of physical pain is one of the primary reasons reported for opioid misuse, which points to the need for improved pain management strategies [6].

\section{CYP2D6 genotype \& opioid response}

Tramadol, codeine, hydrocodone and oxycodone are among the most commonly prescribed opioids in the USA [7]. Each is converted by CYP2D6 to a metabolite with more potent opioid effects than the parent compound. Codeine and tramadol are metabolized to morphine and des-methyltramadol, respectively, which have approximately 200fold greater affinity for the $m u$-opioid receptor than the parent compounds [8]. Hydrocodone is metabolized to hydromorphone and oxycodone to oxymorphone, with approximately 10-30-fold and 40-fold greater affinity, respectively, than their parent compounds [8].

The gene-encoding CYP2D6 is highly polymorphic. Approximately $5-10 \%$ of individuals have two no function CYP2D6 alleles (e.g., ${ }^{*} 4 /^{*} 5$ genotype) resulting in complete absence of enzyme activity and the CYP2D6 poor metabolizer (PM) phenotype [8]. PMs cannot convert codeine, tramadol, hydrocodone or oxycodone to their more potent metabolites and may not attain pain relief from codeine and tramadol, and possibly also from hydrocodone. The data with oxycodone are less clear. An additional $2-11 \%$ of individuals have a no function allele in combination with a reduced function allele (e.g., *41, *17), very little enzyme activity and the intermediate metabolizer (IM) phenotype [8]. The effectiveness of opioids metabolized by CYP2D6 may also be compromised in these individuals [8,9]. At the opposite end of the spectrum, $1-2 \%$ of individuals are ultrarapid metabolizers (UMs), with multiple copies of functional CYP2D6 alleles and increased enzyme activity. These individuals are at risk for life-threatening toxicity following exposure to these opioids as a normal dose leads to metabolism of parent compounds to excessive amounts of their more potent metabolites via CYP2D6 [8]. Concerns about increased toxicity in UMs have prompted a number of hospitals, including our own, to remove codeine from the formulary. However, tramadol, hydrocodone and oxycodone remain on formulary. 


\section{Opioid-labeling changes \& pharmacogenetic guidelines}

In 2015, the US FDA issued a safety communication warning of risk for toxicity with codeine and tramadol in UMs. In 2017, the agency took a step further to contraindicate the use of these drugs in all children younger than 12 years because of risk related to the UM phenotype. Boxed warnings on the FDA-approved labeling for both drugs also warn against their use after tonsillectomy or adenoidectomy in children ages 12-18 years with risk factors for respiratory depression. The warnings apply to all children even though opioids are expected to be safe and effective for approximately $75 \%$ of children with the normal metabolizer (NM) phenotype. This has led some to propose revision of the boxed warning to state that $C Y P 2 D 6$ genotype may be used to identify children at risk for toxicity or poor response and allow use of codeine and tramadol in NMs for settings outside of adenotonsillectomy [10].

The Clinical Pharmacogenetics Implementation Consortium published guidelines for the use of codeine based on CYP2D6 phenotype [8]. Similar to other CPIC guidelines, they do not provide recommendation for or against genetic testing, but instead provide guidance for interpretation and translation of genotype results. The guidelines specifically recommend against codeine use in UMs because of risk for toxicity and in PMs because of lack of efficacy. The guidelines further state that tramadol is not a good alternative because it is also metabolized by CYP2D6, with similar but weaker language for hydrocodone and oxycodone. For IMs, the guidelines recommend considering an alternative analgesic if patients fail to respond to codeine, and again recommend avoidance of other opioids metabolized via CYP2D6 in this situation.

\section{Phenoconversion with CYP2D6 inhibitors}

A number of commonly prescribed medications are moderate (e.g., duloxetine) to strong (e.g., bupropion, fluoxetine, paroxetine) inhibitors of the CYP2D6 enzyme and can phenoconvert patients who have a genotype associated with the NM phenotype to either IM or PM [11]. As depression and pain are common co-morbidities, these drugs are frequently prescribed to patients who may be treated with opioids. This is especially true for duloxetine, given evidence of its effectiveness in pain management [12]. Thus, phenoconversion is an important consideration when selecting opioid therapy regardless of whether or not genotype information is available.

\section{Clinical implementation of CYP2D6 genotyping}

CYP2D 6 genotyping is entering clinical practice at multiple institutions to guide prescribing of opioids, [13] and data are emerging on the feasibility and outcomes with this approach to pain management $[14,15]$. In a recent pragmatic pilot study of CYP2D6 phenotype-guided management of patients with at least 3 months of chronic pain, we found that among PMs or IMs prescribed tramadol or codeine at baseline, there was a significant improvement in pain control with a genotype-guided approach versus traditional pain management [15]. In the genotype-guided arm, CYP2D6 phenotype was assigned based on genotype and use of CYP2D6 inhibitors. Consideration of phenoconversion increased the percent of patients with a PM or IM phenotype from 10\% (based on genotype alone) to 35\% (based on genotype plus use of CYP2D6 inhibitors). Not unexpectedly, the primary drugs causing phenoconversion were duloxetine and bupropion. Pharmacists provided recommendations to physicians to avoid prescribing codeine or tramadol, and to a lesser extent, hydrocodone or oxycodone, to PMs and IMs. Interestingly, when physicians changed therapy based on these recommendations, they were most likely to switch to a nonopioid analgesic.

\section{On-going \& planned clinical trials}

One of the major challenges with pharmacogenetic implementation is having genotype results available at the time a prescribing decision is being made [16]. One approach to address this is to order genotyping preemptively for patients who are anticipated to need a drug in the future. Such an approach is possible with CYP2D6 genotyping for patients scheduled to undergo surgical procedures where significant postoperative pain is expected. In this situation, the genetic sample may be obtained at the time the patient is scheduled for surgery, so that results are returned prior to postoperative opioid prescribing for acute pain management. Our group is conducting a pilot trial as part of our learning health system to investigate the feasibility of preemptive CYP2D6 genotyping for patients scheduled to undergo arthroplastic procedures (Clinical Trials.gov Identifier: NC03534063). Findings from this trial, in addition to our recent findings from our pilot trial of CYP2D6-guided management of chronic pain [15] will inform larger, multisite trials of the efficacy of CYP2D6-guided opioid prescribing for both acute (postoperative) and chronic pain management as part of the Implementing GeNomics In pracTicE (IGNITE) network. 


\section{Future perspective}

CYP2D6 PMs (and to lesser extent IMs) will not derive analgesic effects with codeine or tramadol via the muopioid receptor pathway, and when they indicate that they have poor control with these agents, in the current climate, they may be labeled as drug seeking. On the other hand, use of opioids metabolized via CYP2D6 can have life-threatening consequences in UMs. Thus, we posit that use of codeine and tramadol in absence of CYP2D6 genotype data leads to improper pain management and increases risk for harm.

A CYP2D6-guided approach to pain management, with avoidance of codeine and tramadol in PMs and IMs, can improve pain control, as we showed in our chronic pain study [15]. Moreover, we found that when physicians changed therapy in response to CYP2D6-based recommendations, it was most often to a nonopioid, thus reducing opioid exposure. These data suggest that CYP2D6-guided pain management may have the added benefit of addressing the opioid epidemic, particularly since seeking pain relief is the most commonly cited reason for opioid misuse [6].

For patients with the CYP2D6 NM phenotype, we suggest that tramadol be the preferred opioid because of its dual mechanism of action, with inhibitory effects on norepinephrine and serotonin reuptake in addition to its effects on the mu-opioid receptor, and evidence of its lower addiction potential compared with hydrocodone [17]. This is the approach that we are taking in our on-going study in patients undergoing arthroplastic surgery, and is consistent with the multimodal opioid sparing strategy of the enhanced recovery after surgery pathway, in which primary use of nonopioid agents and techniques and tramadol as the preferred opioid is recommended [18]. Since postoperative opioid prescribing is a major gateway to persistent opioid use [19], use of tramadol in NMs in lieu of highly addictive opioids, like hydrocodone and oxycodone, may also be a mechanism to curb persistent opioid use. Ultimately, incorporating CYP2D6 genotyping into pain management decisions may allow individualized prescribing of pain medications and result in safer and more effective pain management, while also reducing overall opioid exposure.

Financial \& competing interests disclosure

Dr Cavallari has received research funding from Mallinckrodt Pharmaceuticals. This work is supported by NIH/NHGRI (U01HG 007269) and NIH/NCATS (UL1TR001427). The content is solely the responsibility of the authors and does not necessarily represent the official views of the NIH. The authors have no other relevant affiliations or financial involvement with any organization or entity with a financial interest in or financial conflict with the subject matter or materials discussed in the manuscript apart from those disclosed.

No writing assistance was utilized in the production of this manuscript.

\section{References}

1 Blyth FM, Huckel Schneider C. Global burden of pain and global pain policy-creating a purposeful body of evidence. Pain 159(Suppl. 1), S43-S48 (2018).

2 Dahlhamer J, Lucas J, Zelaya C et al. Prevalence of chronic pain and high-impact chronic pain among adults - United States, 2016. MMWR Morb. Mortal. Wkly Rep. 67(36), 1001-1006 (2018).

3 Gureje O, Von Korff M, Simon GE, Gater R. Persistent pain and well-being: a World Health Organization study in primary care. JAMA 280(2), 147-151 (1998).

4 Levy B, Paulozzi L, Mack KA, Jones CM. Trends in opioid analgesic-prescribing rates by specialty, U.S., 2007-2012. Am. J. Prev. Med. 49(3), 409-413 (2015).

5 Centers for Disease Control. Opioid overdose. prescription opioid data. www.cdc.gov/drugoverdose/data/prescribing.html

6 Han B, Compton WM, Blanco C et al. Prescription opioid use, misuse, and use disorders in U.S. adults: 2015 national survey on drug use and health. Ann. Intern. Med. 167(5), 293-301 (2017).

7 IMS Institute for Healthcare Informatics. Medication use and the shifting cost of healthcare - a review of use of medicines in the United States in 2013 (2014). https://democrats-oversight.house.gov/sites/democrats.oversight.house.gov/files/documents/IMS-Medicine $\% 20$ use $\% 20$ and $\% 20$ shif ting $\% 20$ cost $\% 20$ of $\% 20$ healthcare.pdf

8 Crews KR, Gaedigk A, Dunnenberger HM et al. Clinical Pharmacogenetics Implementation Consortium guidelines for cytochrome P450 2D6 genotype and codeine therapy: 2014 update. Clin. Pharmacol. Ther. 95(4), 376-382 (2014).

9 Poulsen L, Arendt-Nielsen L, Brosen K, Sindrup SH. The hypoalgesic effect of tramadol in relation to CYP2D6. Clin. Pharmacol. Ther. 60(6), 636-644 (1996).

10 Gammal RS, Caudle KE, Quinn CT et al. The case for pharmacogenetics-guided prescribing of codeine in children. Clin. Pharmacol. Ther.105(6), 1300-1302 (2019). 
11 Borges S, Desta Z, Jin Y et al. Composite functional genetic and comedication CYP2D6 activity score in predicting tamoxifen drug exposure among breast cancer patients. J. Clin. Pharmacol. 50(4), 450-458 (2010).

12 Schukro RP, Oehmke MJ, Geroldinger A et al. Efficacy of duloxetine in chronic low back pain with a neuropathic component: a randomized, double-blind, placebo-controlled crossover trial. Anesthesiology 124(1), 150-158 (2016).

13 Cavallari LH, Van Driest SL, Prows CA et al. Multi-site investigation of strategies for the clinical implementation of CYP2D6 genotyping to guide drug prescribing. Genet. Med. doi:10.1038/s41436-019-0484-3 (2019) (Epub ahead of print).

14 Gammal RS, Crews KR, Haidar CE et al. Pharmacogenetics for safe codeine use in sickle cell disease. Pediatrics 138(1), pii:e20153479 (2016).

15 Smith DM, Weitzel KW, Elsey AR et al. CYP2D6-guided opioid therapy improves pain control in CYP2D6 intermediate and poor metabolizers: a pragmatic clinical trial. Genet. Med. 21(8), 1842-1850 ( 2019).

16 Cicali EJ, Weitzel KW, Elsey AR et al. Challenges and lessons learned from clinical pharmacogenetic implementation of multiple gene-drug pairs across ambulatory care settings. Genet. Med. doi:10.1038/s41436-019-0500-7 (2019) (Epub ahead of print).

17 Adams EH, Breiner S, Cicero TJ et al. A comparison of the abuse liability of tramadol, NSAIDs, and hydrocodone in patients with chronic pain. J. Pain Symptom. Manage. 31(5), 465-476 (2006).

18 Wick EC, Grant MC, Wu CL. Postoperative multimodal analgesia pain management with nonopioid analgesics and techniques: a review. JAMA Surg. 152(7), 691-697 (2017).

19 Brummett CM, Waljee JF, Goesling J et al. New persistent opioid use after minor and major surgical procedures in US adults. JAMA Surg. 152(6), e170504 (2017). 